\title{
Gauge Contradictions in the QED Lagrangian Density
}

\author{
Eliahu Comay \\ Charactell Ltd., Tel-Aviv, Israel \\ Email: elicomay@post.tau.ac.il
}

How to cite this paper: Comay, E. (2017) Gauge Contradictions in the QED Lagrangian Density. Open Access Library Journal, 4: e3596.

https://doi.org/10.4236/oalib.1103596

Received: April 12, 2017

Accepted: May 13, 2017

Published: May 16, 2017

Copyright $\odot 2017$ by author and Open Access Library Inc.

This work is licensed under the Creative Commons Attribution International License (CC BY 4.0).

http://creativecommons.org/licenses/by/4.0/

\begin{abstract}
This work distinguishes between classical electrodynamics where Maxwell equations and the Lorentz force are used as the theory's cornerstone (MLE) and electrodynamic theories that are derived from the variational principle (VE). The paper explains the significance of this distinction. Mathematical elements of gauge transformations are examined within the realm of these theories. The analysis proves that MLE is a gauge invariant theory whereas errors arise from the introduction of gauge transformations into VE. The paper explains why MLE evades the contradictions of VE.
\end{abstract}

\section{Subject Areas}

Theoretical Physics

\section{Keywords}

Quantum Electrodynamics, Lagrangian Density, Gauge Transformation, Double Slit Experiment

\section{Introduction}

It is well known that classical electrodynamics and quantum electrodynamics (QED) are distinct (but related) theories that refer to different aspects of electromagnetic interactions. However, the entire electromagnetic theory can be divided differently. Thus, classical electrodynamics can be constructed on the assumption where Maxwell equations and the Lorentz force are used as the theory's cornerstone. These differential equations (called herein MLE) depend on Maxwellian fields $F^{\mu \nu}$ and are independent of the 4-potential $A_{\mu}$. In MLE, one may use an arbitrary gauge function $\Lambda(x)$, where $x$ denotes the four space-time coordinates. Evidently, in MLE, an addition of a gauge term $\Lambda(x)_{, \mu}$ to the 4-potential $A_{\mu}$ does not alter the electromagnetic fields $F^{\mu \nu}$ 


$$
F_{\mu v}^{\prime}=A_{v, \mu}-A_{\mu, v}+\Lambda(x)_{, v, \mu}-\Lambda(x)_{, \mu, v}=F_{\mu \nu},
$$

where $F_{\mu \nu}^{\prime}$ denotes the gauge-transformed electromagnetic field. Hence, MLE is a gauge invariant theory.

Alternatively, one may accept a different point of view and take the variational principle as a more fundamental element of electrodynamics (called below VE). Here one can use an appropriate Lagrangian function as the theory's cornerstone and construct both classical electrodynamics [1] and QED [2] [3]. Acceptable theories of VE must obey constraints whose existence is not self-evident in the approach which yields MLE. Thus, in VE the equations of motion must be derived from a Lagrangian density which is a Lorentz scalar whose dimension is $\left[L^{-4}\right]$, where $L$ denotes length. Such a Lagrangian density yields an action which is a dimensionless Lorentz scalar. This kind of action is suitable for the definition of the quantum particle's phase (see e.g. [4], p. 20). The actual form of the electromagnetic Lagrangian depends explicitly of the fields $F^{\mu v}$ and on the 4-potential $A_{\mu}$. Here a gauge transformation alters the 4-potentials which explicitly appear in the theory's cornerstone. Therefore, unlike the case of MLE, the issue of whether or not VE is gauge invariant requires a further examination. The present work is dedicated to an analysis of this problem in the quantum domain.

Electrodynamics is an important sector of physics and it uses extensively gauge transformations. In particular, gauge transformations play a crucial role in the present structure of QED (see. e.g. [2], chapter 8). These issues indicate the relevance of the analysis presented in this work.

An important element of the analysis is the close relationships between quantum mechanics and quantum field theory (QFT). This issue is stated clearly in S. Weinberg's well known textbook: "First, some good news: quantum field theory is based on the same quantum mechanics that was invented by Schroedinger, Heisenberg, Pauli, Born, and others in 1925-26, and has been used ever since in atomic, molecular, nuclear and condensed matter physics" (see [2], p. 49). The term Weinberg correspondence principle is used below for a description of this relationship.

In this work relativistic and quantum expressions are written in the standard notation. The diagonal metric is $(1,-1,-1,-1)$ and units where $\hbar=c=1$ are used. In these units there is just one kind of dimension and it is denoted by an appropriate power of length $\left[L^{n}\right]$. The second section describes several inconsistencies between VE and gauge transformation. The third section shows an example of an interference experiment where VE is inconsistent with the gauge freedom. A discussion of the results is presented in the fourth section. The last section contains concluding remarks.

\section{Problems with Gauge Transformations}

The Lagrangian density of a Dirac electron and electromagnetic fields is the primary QED expression. It takes the following form 


$$
\mathcal{L}=\bar{\psi}\left[\gamma^{\mu} i \partial_{\mu}-m\right] \psi-\frac{1}{16 \pi} F^{\mu v} F_{\mu v}-e \bar{\psi} \gamma^{\mu} A_{\mu} \psi
$$

The first term of (2) represents a free Dirac particle, the second term represents free electromagnetic fields and the last term represents the interaction between the Dirac charged particle and the electromagnetic fields (see [3], p. 78, [5], p. 84).

The gauge transformation affects the electromagnetic 4-potential $A_{\mu}$ and the Dirac function $\psi$. It takes the following form

$$
A_{\mu}(x) \rightarrow A_{\mu}(x)+\Lambda(x)_{, \mu} ; \quad \psi(x) \rightarrow \exp (\operatorname{ie\Lambda }(x)) \psi(x),
$$

(see [2], p. 345, [3], p. 78). An important element of the following analysis is the fact that the gauge function $\Lambda(x)$ is an arbitrary function of $x$ (see [2], p. 342 after (8.1.13); [3], p. 482, after (15.1); [6], p. 207 after (23b); [7], p. 192; [8], p. 316 , after (14.21)). The arbitrariness of the gauge function $\Lambda(x)$ means that it may take different values at different space-time points. This feature of the gauge transformation (3) means that the phase of the Dirac particle changes locally under a gauge transformation (see [3], p. 78).

A straightforward examination proves that the Dirac Lagrangian density (2) is invariant under the gauge transformation (3) because the contributions of the two additional terms cancel each other. This outcome is certainly a necessary condition for gauge invariance of the theory. However, there is no doubt that an acceptable gauge transformations should also satisfy another requirement: it must take a mathematically consistent form. The rest of this work presents several arguments which prove that gauge transformations are unacceptable in QED because of their inconsistent structure.

1) Dimensions of Terms

It is very well known that if $A+B$ is a physically meaningful expression then $A$ and $B$ must have the same dimensions. The gauge transformation (3) shows that it adds an exponential factor to the Dirac function $\psi(x)$. The power series expansion of this exponent is

$$
\exp (i e \Lambda(x))=1+i e \Lambda(x)+\cdots
$$

Evidently, the number 1 which stands on the right hand side of (4) is a dimensionless pure number. It follows that also ie $\Lambda(x)$ must be dimensionless. The pure imaginary number $i$ is dimensionless and in the unit system used herein also the electric charge is a dimensionless number $e^{2} \simeq 1 / 137$.

Conclusion \#1: The gauge function $\Lambda(x)$ must be dimensionless. Therefore, the arbitrariness of the gauge function violates this requirement.

The same result is obtained from the first term of (3). Here the dimension of the 4-potential is $\left[L^{-1}\right]$. Therefore, the arbitrariness of the gauge function violates also this requirement.

2) Lorentz Covariance.

Conclusion \#2: An analogous analysis of (4) proves that the gauge function must also be a Lorentz scalar. Therefore, the arbitrariness of the gauge function 
violates the second requirement.

\section{3) A Logical Contradiction}

The following arguments show how a contradiction arises from the gauge transformation (3) of the Lagrangian density (2).

a) Let us examine the quite simple state of one electron which moves inertially parallel to the $\mathrm{z}$-axis in a region of space where the external fields vanish. Here all physical properties belong to the electron.

b) The combined effect of the two terms of (3) is a gauge transformation. As stated above, it keeps the Dirac Lagrangian density $\mathcal{L}$ invariant.

c) The function $\psi(x)$ of (3) has an undulating factor called phase. For the free electron considered herein, it takes the following exponential form

$$
\psi(x)=\exp [i(k z-\omega t)] \chi \text {. }
$$

Here $k, \omega$ represent the electron's momentum and energy, respectively and $\chi$ denotes a Dirac spinor (see [9], p. 30). In this case the following relation holds (see [9], p. 7)

$$
\omega^{2}=k^{2}+m^{2} .
$$

d) The added exponential factor of (3) means that the gauge transformation changes the phase of $\psi$. Furthermore, due to the arbitrariness of the gauge function $\Lambda(x)$, the change of phase is not uniform and it may take different value at different space-time points.

e) The system considered herein comprises only one electron whose energy and momentum are defined appropriately. It follows that the electron's energy and momentum should not change under a gauge transformation.

f) If the energy and the momentum do not change under a gauge transformation then the de Broglie principle (see [4], pp. 48, 49, [10], pp. 119, 120) says that the phase of $\psi$ should not change (except by an irrelevant global constant factor).

j) The Weinberg correspondence principle proves that this conclusion holds also for QED.

Conclusion \#3: The forgoing points prove that a contradiction arises from the gauge transformation (3). Indeed, it is shown in item 4 that in order to keep the Lagrangian density invariant, the gauge transformation (3) changes the phase of $\psi$. By contrast, items 5 - 7 prove that under a gauge transformation the phase of $\psi$ should not change!

\section{An Interference Example}

An important element of the gauge transformation (3) is the change of the quantum particle's phase. Therefore, one kind of test of gauge transformation compatibility is an examination of an interference experiment where the particle's phase plays a crucial role. Here the calculations adhere to the inherent gauge transformation property where the gauge function is an arbitrary function of the four space-time coordinates. 
Consider an electron that moves parallel to the $z$-axis. The external fields vanish and the electron moves inertially. The electron's wave function takes the form of (5) and in the experiment discussed herein, the components of the electron's momentum are

$$
k_{x}=k_{y}=0, k_{z}=1 .
$$

It passes through two slits and the two sub-beams interfere on the screen $S$ (see Figure 1). The sine curve of Figure 1 represents the intensity of the constructive/destructive interference at the corresponding points on $S$ (see [11], p. 239). This curve is obtained from a straightforward calculation of the phase of the two sub-beams.

Let us examine how the following gauge transformation

$$
\Lambda(x)=-z / e
$$

affects the interference calculation. Here the gauge function depends only on the $z$-coordinate. Using the specific value $k_{z}=1$, one finds that a substitution of the electron's function (5) and the gauge function (8) into the gauge transformation (3) yields the following wave function

$$
\psi(x)=\exp (i(z-\omega t)) \chi \rightarrow \exp (-i \omega t) \chi .
$$

Evidently, the $z$-dependence of the gauge function (8) cancels the $z$-dependence of the original electron's phase. The final outcome of (9) is a wave that has a uniform spatial dependence. It follows that there is no destructive interference and the interference pattern of Figure 1 is destroyed.

Section 2 presents several theoretical inconsistencies of gauge transformations in VE. The contradiction obtained in this section provides a further support for these inconsistencies. It examines a specific case where the gauge transformed function (9) violates the de Broglie relation between the particle's phase and its energy-momentum values (6).
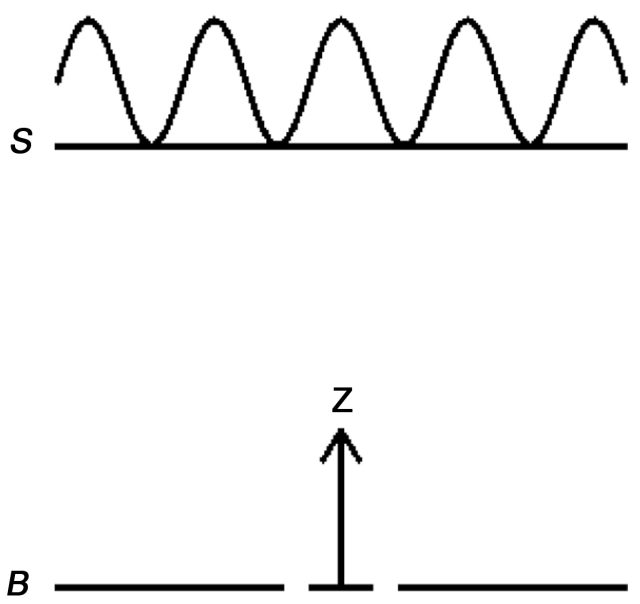

Figure 1. An electronic beam moves parallel to the $\mathrm{z}$-axis. The beam hits the barrier $\mathrm{B}$ and two sub-beams pass through the slits. Later, the subbeams interfere on the screen $S$ (see text). 


\section{Discussion}

The present work distinguishes between the theoretical structure of MLE and that of VE. Certainly, MLE is a gauge invariant theory because Maxwell equations and the Lorentz force are independent of the 4-potential which varies under a gauge transformation. The present work discusses the Lagrangian density of QED which is a quantum theory derived from VE. Evidently, MLE is a classical theory where the particle's phase has no meaning. By contrast, the particle's phase is a cardinal element of a quantum theory. The Weinberg correspondence principle means that this property also holds for QED. This work analyzes effects of gauge transformation on the particle's phase (3). The analysis adheres to the well known attribute of the gauge function which is an arbitrary function of the four space-time coordinates. Section 2 presents three different theoretical contradictions of gauge transformations and section 3 describes a specific case where a gauge transformation yields unacceptable results.

The discussion presented above shows how errors emerge from the second term of the gauge transformation (3). The fact that the Lagrangian density is invariant under the two terms of (3) indicates that also the first term of (3) is likely to be erroneous. And indeed, an analysis of the first term of (3) proves that it yields errors [12]. This effect is also found in the classical domain of VE. For example, one examines a free motionless electron and introduces the gauge transformation function $\Lambda(x)=t^{2}$ into (3). Here the null 4-potential transforms as follows

$$
A_{\mu}(x)=(0,0,0,0) \rightarrow A_{\mu}(x)=(2 t, 0,0,0) .
$$

Under this transformation the Hamiltonian of the free motionless electron discussed herein boils down to the following time-dependent expression (see [1], p. 49)

$$
H=m+2 e t .
$$

The explicit time-dependence of the Hamiltonian means that in the VE version of classical electrodynamics a gauge transformation violates energy conservation.

Section 2 proves the dimension inconsistency of gauge transformations as well as their failure to satisfy covariance. These properties mean that the success of gauge transformations in MLE is simply because here the gauge errors of (1) take the form of a 4-curl and the difference between the two terms of this 4-curl vanishes. Therefore, in MLE an addition of a null value that has the wrong dimension is apparently acceptable.

\section{Conclusions}

The primary element of this work is the distinction between the theoretical structure of Maxwell-Lorentz electrodynamics (MLE) and that of variational electrodynamics (VE). It presents theoretical proofs of gauge transformations unacceptability in VE as well as an example of contradictory results of a specific interference experiment. It also explains that in MLE gauge transformations look 
OK just because here the 4-curl of the gauge function vanishes.

Gauge transformations play an important role in the present structure of QED (see [2], pp. 339-343). For this reason, the above mentioned gauge transformations errors point out the need to reconstruct QED in a consistent form. The recent experimental QED failure to explain the proton charge radius data [13] can be regarded as an experimental support for the theoretical QED problems which are proved in this work.

\section{References}

[1] Landau, L.D. and Lifshitz, E.M. (2005) The Classical Theory of Fields. Elsevier, Amsterdam.

[2] Weinberg, S. (1995) The Quantum Theory of Fields. Vol. 1, Cambridge University Press, Cambridge.

[3] Peskin, M.E. and Schroeder, D.V. (1995) An Introduction to Quantum Field Theory. Addison-Wesley, Reading.

[4] Landau, L.D. and Lifshitz, E.M. (1959) Quantum Mechanics. Pergamon, London.

[5] Bjorken, J.D. and Drell, S.D. (1965) Relativistic Quantum Fields. McGraw-Hill, New York.

[6] Pauli, W. (1941) Relativistic Field Theories of Elementary Particles. Reviews of Modern Physics, 13, 203.

[7] Yang, C.N. and Mills, R. (1954) Conservation of Isotopic Spin and Isotopic Gauge Invariance. Physical Review, 96, 191.

[8] Halzen, F. and Martin, A.D. (1984) Quarks and Leptons: An Introductory Course in Modern Particle Physics. John Wiley, New York.

[9] Bjorken, J.D. and Drell, S.D. (1964) Relativistic Quantum Mechanics. McGraw-Hill, New York.

[10] Dirac, P.A.M. (1958) The Principles of Quantum Mechanics. 4th Edition, Oxford University Press, Oxford.

[11] Jenkins, F.A. and White, H.E. (1957) Fundamentals of Optics. McGraw-Hill, New York.

[12] Comay, E. (2008) Mathematical Constraints on Gauge in Maxwellian Electrodynamics. Apeiron, 15, 123.

[13] Pohl, R., et al. (2010) The Size of the Proton. Nature, 466, 213. 
Submit or recommend next manuscript to OALib Journal and we will provide best service for you:

- Publication frequency: Monthly

- 9 subject areas of science, technology and medicine

- Fair and rigorous peer-review system

- Fast publication process

- Article promotion in various social networking sites (LinkedIn, Facebook, Twitter, etc.)

- Maximum dissemination of your research work

Submit Your Paper Online: Click Here to Submit

Or Contact service@oalib.com 